\title{
A combined study of mesomorphism, optical, and electronic properties of donor-acceptor columnar liquid crystals
}

\author{
S. Holger Eichhorn*a ${ }^{\mathrm{a}}$, Chen Shuai ${ }^{\mathrm{a}}$, Mohamed Ahmida ${ }^{\mathrm{a}}$, Andrey Demenev ${ }^{\mathrm{a}}$, Himadri Kayal ${ }^{\mathrm{a}}$, Farah \\ S. Raad ${ }^{\mathrm{a}, \mathrm{b}}$, Bilal R. Kaafarani ${ }^{\mathrm{b}}$, Sameer Patwardhan ${ }^{\mathrm{c}}$, Ferdinand C. Grozema ${ }^{\mathrm{c}}$, Laurens D. A. \\ Siebbeles ${ }^{\mathrm{c}}$, Tyler Taerum ${ }^{\mathrm{d}}$, Dmitrii F. Perepichka ${ }^{\mathrm{d}}$, Richard Klenkler ${ }^{\mathrm{e}}$ \\ ${ }^{a}$ Department of Chemistry \& Biochemistry, University of Windsor, 401 Sunset Avenue, Windsor, \\ ON, Canada N9B 3P4; \\ ${ }^{b}$ Department of Chemistry, American University of Beirut, Beirut 1107-2020, Lebanon; \\ 'DelftChemTech, Delft University of Technology, Julianalaan 136, 2628 BL Delft, The Netherlands; \\ ${ }^{\mathrm{d}}$ Department of Chemistry, McGill University, 801 Sherbrooke Street West, Montreal, QC, Canada \\ H3A 2K6; \\ ${ }^{\mathrm{e} X e r o x}$ Research Centre Canada, 2660 Speakman Drive, Mississauga, ON, Canada L5K 2L1
}

\begin{abstract}
Donor-acceptor structures have recently gained great popularity for the design of low band gap polymeric organic semiconductors. Presented here is a first systematic study of organic semiconductors based on columnar liquid crystals that consist of discotic and board-shaped donor-acceptor structures. The discotic benzotristhiophenetricarboxamide and hexaphenyldiquinoxalinophenazine derivatives form hexagonal columnar mesophases over wide temperature ranges while the board-shaped tetraphenylquinoxalinophenanthrophenazine derivative displays rectangular and hexagonal columnar mesophases. All compounds are designed to preferentially conduct electrons and not holes because the central acceptor parts, which are the sole contributors to the unoccupied frontier orbitals, show strong intermolecular electronic interactions within columnar stacks whereas the donor parts at the periphery of the cores, the sole contributors to the occupied frontier orbitals, are too far apart to provide sufficient electronic interactions. The absence of hole conduction is confirmed by charge carrier mobility measurements based on time-resolved microwave conductivity and time-of-flight methods that reveal intrinsic electron mobility values of about $10^{-2} \mathrm{~cm}^{2} \mathrm{~V}^{-1} \mathrm{~s}^{-1}$. The board-shaped compound is also highly fluorescent not only in solution but also in its mesophases. Interstingly, the fluorescence quantum yield of its mesophases reversibly increases with decreasing temperature, which is reasoned with changes in molecular mobility and intracolumnar packing within columnar stacks.
\end{abstract}

Keywords: Discotic, columnar, liquid crystals, charge carrier mobility, donor-acceptor, fluorescence, H-bonding, electron conductors

\section{INTRODUCTION}

Self-organized columnar stacks of aromatic compounds such as discotic liquid crystals (DLCs) ${ }^{1}$ and polyaromatic dendrons $^{2}$ are an alternative class of organic semiconductors that may show charge carrier mobility values above 1 $\mathrm{cm}^{2} \mathrm{~V}^{-1} \mathrm{~s}^{-1}$ along the columnar stacks. ${ }^{3,4,5}$ In addition to efficient charge transport and the advantages generic to organic semiconductors, such as low temperature solution processing and synthetically adjustable properties, DLCs promise an important capability of self-healing and can be used in the formation of highly anisotropic materials.

Two important limitations have hampered a broader application of columnar liquid crystalline materials for organic electronics: 1. the challenging formation of aligned monodomains of the required orientation and size; 2 . the complexity of their molecular design that must consider optical and electronic properties as well as mesomorphism and stability. Design criteria must control not only molecular properties, such as electron affinity and oxidation potential, electronic excitation energies, as well as chemical and thermal stability, but also intermolecular interactions and self-organization.

Demonstrated here is the application of new donor-acceptor structures for the design of electron conducting and fluorescent columnar liquid crystals. Charge carrier mobility was measured by time resolved microwave conductivity (TRMC) that provides intrinsic combined mobility values and time-of-flight photoconductivity that provides mobility

Liquid Crystals XV, edited by lam Choon Khoo, Proc. of SPIE Vol. 8114, 811402

(c) 2011 SPIE · CCC code: $0277-786 \mathrm{X} / 11 / \$ 18 \cdot$ doi: $10.1117 / 12.893149$ 
values for electrons and holes. ${ }^{1,6}$ The obtained mobility data are interpreted based on quantum chemical molecular descriptions of the dependence of charge carrier mobility on intermolecular electronic interactions. ${ }^{7,8,9}$ This approach based on the Markus theory predicts an increase in hopping rate (charge carrier mobility) with increasing transfer integral and decreasing reorganization energy. The transfer integral, which is a function of the overlap of frontier orbitals of adjacent molecules, crucially depends on the relative distances, positions, and orientations of stacked aromatic cores.

The application of columnar organic semiconductors as light emitting materials in devices such as OLEDs is hampered by low fluorescence quantum yields because the co-facial stacking in columns often generates $\mathrm{H}$-aggregates. Their lowest exited state is not optically coupled to the ground state, which results in a low fluorescence quantum yield. A potential remedy is to design molecules that form columnar $\pi-\pi$ stacks in which the parallel alignment of transition dipoles in columnar stacks is disfavoured by packing constrains. In fact, an orthogonal orientation of transition dipoles of stacked chromophores should result in a minimum coupling between their transition dipoles. ${ }^{10,11}$ Similarly, board-shaped perylene derivatives have been shown to form fluorescent columnar mesophases when they arrange into tilted stacks (Jaggregates). ${ }^{12,13}$

*eichhorn@uwindsor.ca; phone 1519 253-3000 x3990; fax 1519 973-7098;

http://cronus.uwindsor.ca/users/e/eichhorn/main.nsf

\section{RESULTS AND DISCUSSION}

\subsection{Donor-Acceptor Compounds}

All three compounds N,N',N"'-(3,4,5-tridodecyloxyphenyl)benzo[b,b',b']tristhiophene-2,2',2"'-tricarboxamide $(\mathrm{TTT})^{14}, 2,3,8,9,14,15$-hexa(3,4-didodecyloxyphenyl)diquinoxalino[2,3-a:2',3'-c]phenazine (DQP), and 9,18-bis(1,1dimethylethyl)-4,5,13,14-tetra(3,4-dodecyloxyphenyl)quinoxalino[2',3':9,10]phenanthro[4,5-abc]phenazine (QPP) contain tris- or dialkoxyphenyl groups as electron rich (donor) parts and relatively electron deficient central core structures (Scheme 1). The dodecyloxyphenyl groups are not co-planar with the central core because of steric reasons but the phenyl rings are considered to be a part of the central core because they are rather rigidly connected to the inner cores and dictate the stacking geometry of the cores within the columnar mesophases.

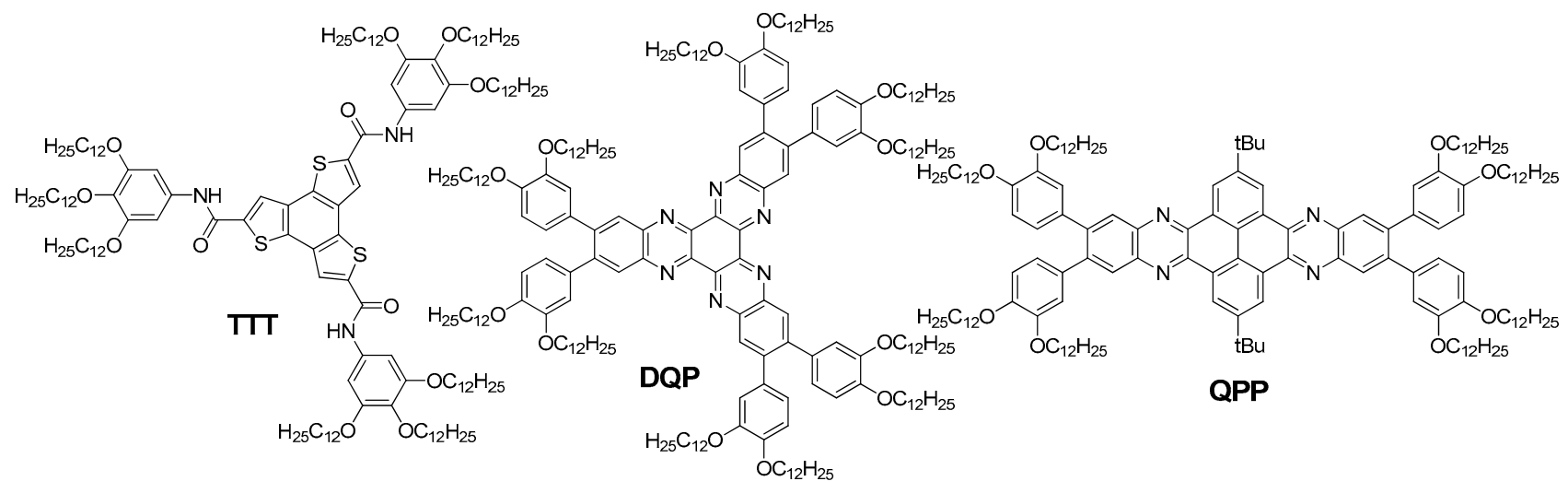

Scheme 1. Molecular structures of donor-acceptor liquid crystals. TTT and DQP are discotic (disc-shaped) while QPP is board-shaped with an aspect ratio of about 6.

\subsection{Properties of TTT}

The disc-shaped TTT expectedly forms a hexagonal columnar mesophase $\left(\mathrm{Col}_{h}\right)$ that freezes into a glassy state below $10{ }^{\circ} \mathrm{C}$ (Fig. 1). Detailed variable temperature IR and XRD studies reveal a helical structure within each columnar stack dictated by intermolecular H-bonds between amide groups of neighboring molecules. Surprisingly, the H-bonded helical structure is persistent over the entire temperature range of the $\mathrm{Col}_{\mathrm{h}}$ up to $286{ }^{\circ} \mathrm{C}$, the transition temperature into the isotropic phase. Both, the lattice parameter of the $\mathrm{Col}_{h}$ phase and the stacking distance increase with increasing 
temperature from $33.9 \AA$ and $3.39 \AA$ at $-25^{\circ} \mathrm{C}$ to $37.5 \AA$ and $3.57 \AA$ at $250{ }^{\circ} \mathrm{C}$, respectively. These changes appear to be linear within the high temperature $\mathrm{Col}_{\mathrm{h}}$ phase, but about $35 \%$ of the total increases occur at the phase transition between the high and low temperature $\mathrm{Col}_{\mathrm{h}}$ phases at about $-10{ }^{\circ} \mathrm{C}$.

Any increase in stacking distance with temperature must be accompanied by a decrease in rotational displacement between adjacent benzotristhiophene units because of the persistent intermolecular $\mathrm{H}$-bonding between amide groups but does not affect much the average distance between the centres of the phenyl rings of about $9 \AA$ (Fig. 1). This is significant because the trialkoxyphenyl groups contribute to $100 \%$ to the highest occupied frontier orbitals of TTT whereas the central benzotristhiophene core contributes $100 \%$ to the lowest unoccupied frontier orbitals (Fig. 1). Recent theoretical studies describe hole and electron transport along columnar stacks via the charge transfer integrals of their occupied and unoccupied frontier orbitals, respectively, and suggest that TTT conducts only electrons because the charge transfer integral between occupied frontier orbitals is close to zero due to the large distance between trialkoxyphenyl groups.

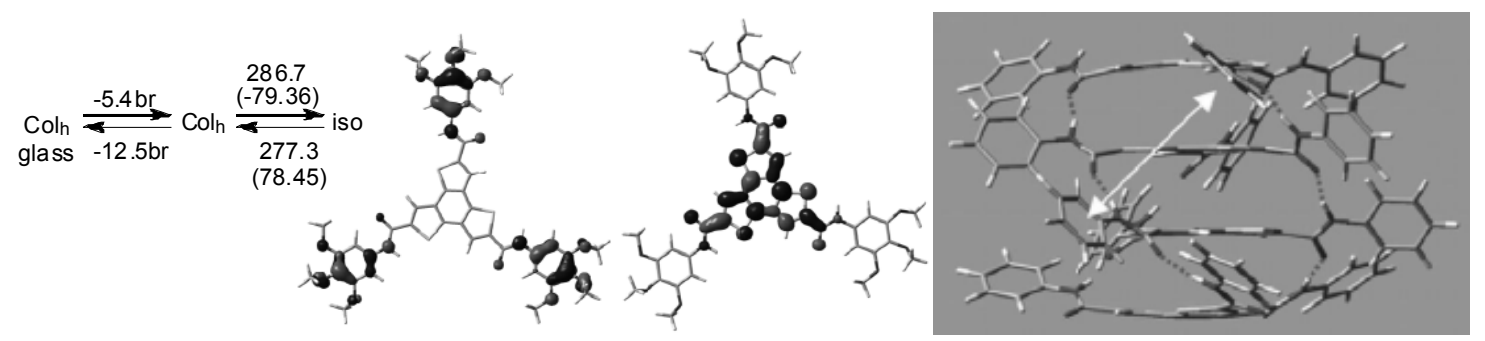

Figure 2. Phase behavior of TTT based on polarized optical microscopy, differential scanning calorimetry, and variable temperature X-ray diffraction (left). Calculated HOMO + HOMO-1 at $-5.51 \mathrm{eV}$, LUMO + LUMO+1 at $2.06 \mathrm{eV}$, and molecular stack of tetramer (centre left to right) (DFT, B3LYP/3-21G*). The white arrow indicates the closest co-facial distance of $9 \AA$ between phenyl rings. ${ }^{14}$

Indeed, the intrinsic combined mobility of $0.02 \mathrm{~cm}^{2} \mathrm{~V}^{-1} \mathrm{~s}^{-1}$ measured by time-resolved microwave conductivity (TRMC) is solely due to electron transport because no transient signal for holes is observed by time-of-flight (TOF) charge carrier mobility measurements. The observed electron mobility is surprisingly high considering that the measurements were taken in air on samples that are not ultra pure and with a compound that has a high $E_{\mathrm{LUMO}}$ of about $-2.3 \mathrm{eV}$.

However, the estimated HOMO-LUMO gap of $3.1 \mathrm{eV}$ remains relatively large despite the donor-acceptor structure of TTT because the inner benzotristhiophene core is a weak electron acceptor. HOMO and LUMO energies are calculated based on cyclic voltammetry (CV) and differential pulse voltammetry (DPV) measurements in solution as well as the optical on-set of absorption in solution and as thin film. A value of $-5.4 \mathrm{eV}$ is obtained for $E_{\text {НОмо }}$ based on $E_{\text {оx }}$ of TTT using ferrocene as internal standard, which is in excellent agreement with the calculated $E_{\text {Номо }}$ of $-5.51 \mathrm{eV}$. The calculated $E_{\text {LUMO }}$ of $-2.06 \mathrm{eV}$ is rather high and could not be measured electrochemically but a value of $-2.3 \mathrm{eV}$ is determined based on $E_{\mathrm{ox}}$ and the optical HOMO-LUMO gap.

\subsection{Properties of DQP}

Mesomorphism and electronic properties of TTT are dominated by the H-bonding interactions between amide groups because they dictate the supramolecular structure of the columnar stacks, which in turn controls electronic interactions and properties of the mesophase. H-bonded columnar mesophases are also interesting model structures because their supramolecular structure is more ordered and less dynamic than supramolecular structures of discotic molecules with weaker intermolecular interactions. However, a potential disadvantage for applications of H-bonded columnar mesophases as organic semiconductors is their difficult alignment on substrates. DQP was prepared and studied to demonstrate that many of the properties of TTT can be achieved without the aid of H-bonds. 


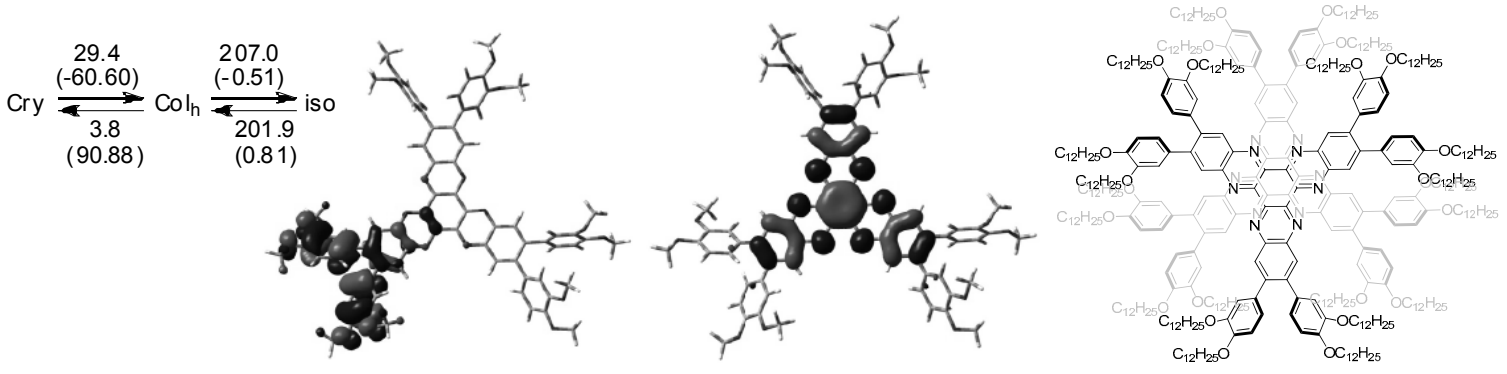

Figure 2. Phase behavior of DQP based on polarized optical microscopy, differential scanning calorimetry, and variable temperature X-ray diffraction (left). Calculated HOMO (-5.32 eV) and LUMO (-2.57 eV) of DQP (DFT, B3LYP/6-31G(d,p)) (centre left and right). Cartoon of two stacking molecules that are rotated with regard to each other by about $60^{\circ}$ to accommodate the non-planar phenyl groups (right).

DQP is a crystalline compound when precipitated from solution below $30^{\circ} \mathrm{C}$ that forms a single $\mathrm{Col}_{\mathrm{h}}$ phase above $30^{\circ} \mathrm{C}$ and clears into an isotropic liquid at $207^{\circ} \mathrm{C}$ (Fig. 2). All transitions are reversible but crystallization occurs at $26^{\circ} \mathrm{C}$ lower temperature than melting. This large hysteresis is not unexpected because the conformationally dynamic and tilted out of planarity phenyl groups complicate the crystallization process. The tilted phenyl groups also dictate a staggered stacking of the cores as illustrated in Fig. 2, which is why $\pi-\pi$ interactions along the columnar stack are persistent only for the central hexaazatriphenylene core but not the phenyl groups. Consequently, no persistent $\pi-\pi$ stacking between the phenyl groups occurs in the columnar stacks of DQP, which suppresses hole conduction because the phenyl groups mainly contribute to the highest occupied frontier orbitals (Fig. 2). DQP predominantly conducts electrons because only the central hexaazatriphenylene core is involved in persistent $\pi-\pi$ stacking and mainly contributes to the lowest unoccupied frontier orbitals. These properties are very similar to the properties of TTT described above but the absent Hbonds result in a less defined and more dynamic supramolecular structure of the columnar stacks of DQP.

The intrinsic combined charge carrier mobility of DQP measured by TRMC is $0.02 \mathrm{~cm}^{2} \mathrm{~V}^{-1} \mathrm{~s}^{-1}$, which is identical with the value obtained for TTT. DQP, however, has a smaller HOMO-LUMO gap than TTT with $2.2 \mathrm{eV}$, which is a result of the much higher electron affinity of the hexaazatriphenylene core in comparison to the benzotristhiophene core as confirmed by CV. The first oxidation potentials of DQP and TTT determined by CV in solution are basically identical while the first reduction potential is over 1 volt lower for DQP. $E_{\text {HOMO }}$ and $E_{\mathrm{LUMO}}$ of DQP can be estimated to $-5.49 \mathrm{eV}$ and $-3.35 \mathrm{eV}$, respectively, based on the $\mathrm{CV}$ data and ferrocene as internal standard.

\subsection{Properties of QPP}

A discrimination between electron and hole conduction in columnar mesophases may also be achieved by deviating from disc-shaped to more board-shaped structures as shown here for QPP. Owing to its different shape anisotropy QPP displays rich polymesomorphism with three columnar mesophases of rectangular symmetry $(\mathrm{Col})$ and a high temperature $\mathrm{Col}_{h}$ phase (Fig. 3). The average stacking distances between the molecules measured by XRD remains surprisingly constant at 3.5 to $3.6 \AA$ across all phase transitions. Computational studies suggest that stacked molecules must be rotated by about $40-60^{\circ}$ with regard to each other to achieve a distance of $3.5 \AA$ because of the repulsion between $t$-butyl groups and tilted out of planarity dialkoxyphenyl rings. In addition to the rotation, molecules are also tilted with regard to the stacking axis (shifted off-centre with regard to each other) in the $\mathrm{Col}_{\mathrm{r}}$ mesophases.

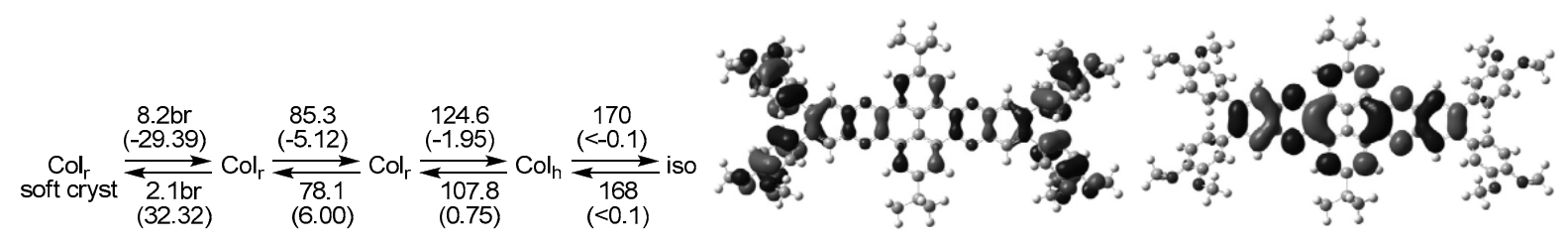

Figure 3. Phase behavior of QPP based on polarized optical microscopy, differential scanning calorimetry, and variable temperature X-ray diffraction (left). Calculated HOMO (-5.46 eV) and LUMO (-3.02 eV) of QPP (DFT, B3LYP/6-31G(d,p)) (centre and right).

Both, a rotation angle of $40-60^{\circ}$ and an off-centred stacking minimizes electronic interactions between the electron rich terphenyl groups that predominantly contribute to the occupied frontier orbitals and biases charge transport towards electron conduction as described above for TTT and DQP. QPP is reversibly reduced at $E_{1 / 2}=-1.29 \mathrm{~V}$ and $E_{1 / 2}=-1.49 \mathrm{~V}$ 
in $\mathrm{CH}_{2} \mathrm{Cl}_{2}$ solution and irreversibly oxidized at $E_{\mathrm{p}}=+1.23 \mathrm{~V}$ and $E_{\mathrm{p}}=+1.48 \mathrm{~V} v \mathrm{Ag} / \mathrm{Ag}^{+}$. Based on the redox potentials in solution and by using ferrocene as reference $E_{\mathrm{HOMO}}$ and $E_{\mathrm{LUMO}}$ are estimated to $-5.46 \mathrm{eV}$ and $-3.02 \mathrm{eV}$, respectively. The calculated electrochemical $E_{\text {gap }}$ of $2.44 \mathrm{eV}$ is large for a donor-acceptor structure, which is mainly due to the relatively weak acceptor properties of the central quinoxalinophenanthrophenazine core.

QPP, in contrast to TTT and DQP, is also strongly fluorescent even in its mesophases and changes in the stacking structure appear to alter fluorescence quantum yields because of changing interactions between transition dipole moments (Fig. 4). The absorption maximum for the lowest energy excitation of QPP is at $445 \mathrm{~nm}$ in $\mathrm{CH}_{2} \mathrm{Cl}_{2}$ solution and its emission maximum is at $549 \mathrm{~nm}$ with a fluorescence quantum yield of $45 \%$ in comparison to Coumarin 6 as standard. The large Stokes shift of $104 \mathrm{~nm}$ suggests that significant structural changes occur after electronic excitation.

A comparison of the absorption and emission spectra in solution and film reveals a distinct red-shift of the longest wavelength absorption maximum $(445 \mathrm{~nm})$ by $7 \mathrm{~nm}$ and a blue-shift of the emission maximum by up to $41 \mathrm{~nm}$ in the films in comparison to the values in $\mathrm{CH}_{2} \mathrm{Cl}_{2}$ solution. These changes indicate strong electronic interactions between molecules in the mesophases but no H-aggregation that would cause a blue-shift of the absorption maxima and a drastic decrease in fluorescence quantum yield.
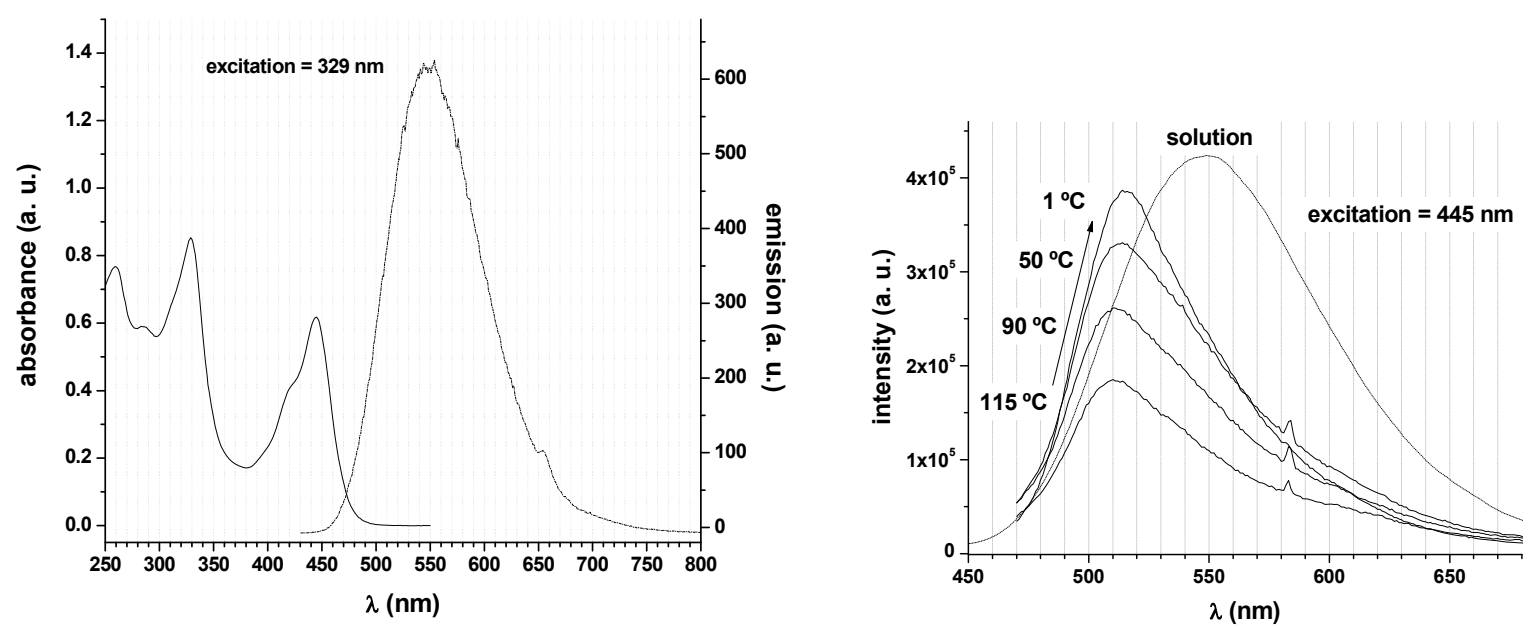

Figure 4. Absorption (solid) and fluorescence (dash-dot) spectra of QPP in $\mathrm{CH}_{2} \mathrm{Cl}_{2}$ solution $\left(8.6 \times 10^{-7} \mathrm{M}\right)$. Excitation at $445 \mathrm{~nm}$ produces an emission spectrum of identical shape and maximum as excitation at $329 \mathrm{~nm}$ (left). Variable temperature fluorescence spectra of QPP as thin film on quartz upon cooling under $\mathrm{N}_{2}$ (solid lines) and in $\mathrm{CH}_{2} \mathrm{Cl}_{2}$ solution at $25^{\circ} \mathrm{C}$ (right).

Remarkably, the fluorescence of QPP remains intense in the columnar mesophases although the fluorescence intensity decreases and the emission maximum blue-shifts by $6 \mathrm{~nm}$ with increasing temperature (Fig. 4). All changes in emission intensity and maxima with temperature are reversible if the measurements are performed under $\mathrm{N}_{2}$ and excitation times are kept short to avoid photobleaching. Absorption spectra of the films changed with temperature to only a small degree and emission spectra are not adjusted for the up to $10 \%$ increase in absorption at the excitation wavelength with increasing temperature. However, a quantitative evaluation of the emission data of the films has not yet been successful.

\section{CONCLUSIONS}

Aromatic donor-acceptor structures are powerful building blocks for organic semiconductors based on columnar mesophases because they can be used not only for lowering absorption wavelengths and HOMO-LUMO energy gaps but also for biasing conduction of charge carriers and altering fluorescence wavelengths and intensities. Both, electron deficient (acceptor) and electron rich (donor) structures are part of the stacking rigid cores but the donor structure does not participate in the $\pi-\pi$ stacking of the central acceptor cores. This is achieved by an out of planarity orientation of the donor structures that induces helical-like stacking within the columns of all three presented compounds TTT, DQP, and QPP and avoids the formation of donor-acceptor stacks due to charge-transfer interactions. The lack of persistent $\pi$ - 
$\pi$ interactions between donor structures along the columnar stacks also suppresses hole conduction because the donor structures contribute predominantly to the highest occupied frontier orbitals. In contrast, the $\pi-\pi$ stacking acceptor cores contribute predominantly to the lowest unoccupied frontier orbitals, which results in high charge transfer integrals for electron conduction. TRMC and TOF charge carrier mobility measurements confirm an intrinsic electron mobility of $0.02 \mathrm{~cm}^{2} \mathrm{~V}^{-1} \mathrm{~s}^{-1}$ for TTT and DQP but no measureable hole mobility.

The combination of donor-acceptor structure and board-shaped core structure exemplified with QPP is also an interesting strategy for the design of fluorescent columnar liquid crystals because fluorescence quenching due to $\mathrm{H}$ aggregation is circumvented if a rotation angle between stacking molecules and/or an off-centered stacking is enforced by the tilted donor parts and a board-shaped structure. These organic semiconductors are of potential use in emitting devices such as OLEDs. Interestingly, the fluorescence intensity of QPP reversibly decreases with increasing temperature of the mesophases, which is explained with temperature dependent average rotation and tilt angles within the columnar mesophases.

\section{ACKNOWLEDGEMENT}

SHE acknowledges supported by NSERC, CFI, OIT, and Xerox and BRK is grateful for support by the Petroleum Research Fund (PRF) of the American Chemical Society (grant \#: 47343-B10) and the Masri Institute of Energy and Natural Resources. F.C.G. acknowledges a VENI grant from the Dutch Foundation for Scientific Research (NWO). T.T. and D.F.P. acknowledge support from NSERC of Canada, Centre for Self-Assembled Chemical Structures and $\mathrm{CFI} / \mathrm{LOF}$.

\section{REFERENCES}

[1] Kumar, S., [Chemistry of Discotic Liquid Crystals], CRC Press, London \& New York, 2010.

[2] Percec, V., Glodde, M., Bera, T. K., Miura, Y., Shiyanovskaya, I., Singer, K. D., Balagurusamy, V. S. K., Heiney, P. A., Schnell, I., Rapp, A., Spiess, H. W., Hudson, S. D., Duan, H., Self-organization of supramolecular helical dendrimers into complex electronic materials," Nature 419(6905), 384-387 (2002).

[3] Laschat, S., Baro, A., Steinke, N., Giesselmann, F., Hagele, C., Scalia, G., Judele, R., Kapatsina, E., Sauer, S., Schreivogel, A., Tosoni, M., "Discotic liquid crystals: From tailor-made synthesis to plastic electronics," Angew. Chem. Int. Edit. 46(26), 4832-4887 (2007).

[4] Sergeyev, S., Pisula, W., Geerts, Y. H., "Discotic liquid crystals: A new generation of organic semiconductors," Chem. Soc. Rev. 36(12), 1902-1929 (2007).

[5] Bushby, R. J., Lozman, O. R., "Photoconducting liquid crystals," Curr. Opin. Solid St. M. 6(6), 569-578 (2002).

[6] Grozema, F. C., Siebbeles, L. D. A., "Mechanism of charge transport in self-organizing organic materials," International Reviews in Physical Chemistry 27(1), 87-138 (2008).

[7] Cornil, J., Lemaur, V., Calbert, J. P., Bredas, J. L., "Charge transport in discotic liquid crystals: A molecular scale description," Adv. Mater., 14(10), 726-729 (2002).

[8] Lemaur, V., Da Silva Filho, D. A., Coropceanu, V., Lehmann, M., Geerts, Y., Piris, J., Debije, M. G., Van de Craats, A. M., Senthilkumar, K., Siebbeles, L. D. A., Warman, J. M., Bredas, J. L., Cornil, J., "Charge transport properties in discotic liquid crystals: A quantum-chemical insight into structure-property relationships," J. Am. Chem. Soc. 126(10), 3271-3279 (2004).

[9] Xinliang, F., Marcon, V., Pisula, W., Hansen, M. R., Kirkpatrick, J., Grozema, F., Andrienko, D., Kremer, K., Müllen, K., "Towards high charge-carrier mobilities by rational design of the shape and periphery of discotics," Nat. Mater. 8(5), 421-426 (2009).

[10] De Halleux, V., Calbert, J. P., Brocorens, P., Cornil, J., Declercq, J. P., Bredas J. L. and Geerts, Y., “1,3,6,8Tetraphenylpyrene Derivatives: Towards Fluorescent Liquid-Crystalline Columns,” Adv. Funct. Mater. 14(7), 649-659 (2004).

[11] Hayer, A., de Halleux, V., Kohler, A., El-Garoughy, A., Meijer, E. W., Barbera, J., Tant, J., Levin, J., Lehmann, M., Gierschner, J., Cornil J. and Geerts, Y. H. "Highly fluorescent crystalline and liquid crystalline columnar phases of pyrene-based structures," J. Phys. Chem. B 110(15), 7653-7659 (2006). 
[12] Sautter, A., Thalacker C. and Würthner, F., "Control of liquid crystallinity of diazadibenzoperylene dyes by covalent and hydrogen-bonded attachment of mesogens," Angew. Chem. Int. Edit. 40(23), 4425-4428 (2001).

[13] Hassheider, T., Benning, S. A., Kitzerow, H. S., Achard M. F. and Bock, H., "Color-Tuned Electroluminescence from Columnar Liquid Crystaline Alkyl Arenecarboxylates,” Angew. Chem. Int. Edit. 40(11), 2060-2063 (2001).

[14] Demenev, A., Eichhorn, S. H., Taerum, T., Perepichka, D. F., Patwardhan, S., Grozema, F. C., Siebbeles, L. D. A., Klenkler, R., "Quasi Temperature Independent Electron Mobility in Hexagonal Columnar Mesophases of an H-Bonded Benzotristhiophene Derivative," Chem. Mater. 22(4), 1420-1428 (2010). 